\title{
Free Migration of Intraocular Glass in Aphakia after Glaucoma Surgery
}

\author{
Dan-dan Zhou ${ }^{a} \quad$ Ling Gao $^{b} \quad K^{2}$ ai-min Guo ${ }^{c}$ Ji-long Hao ${ }^{d}$ \\ Cheng-wei Lu $^{\mathrm{d}}$ \\ Departments of ${ }^{\mathrm{a}}$ Radiology, ${ }^{\mathrm{b}}$ Radiation Oncology, ${ }^{\mathrm{c}}$ Andrology and ${ }^{\mathrm{d} O p h t h a l m o l o g y,}$ \\ The First Hospital of Jilin University, Changchun, China
}

\section{Key Words}

Glass intraocular foreign bodies · Glaucoma surgery · Corneal edema

\begin{abstract}
Purpose: To describe a case of free migration of intraocular glass in aphakia after glaucoma surgery. Methods: We report the case of a 27-year-old man with a history of perforating injury to the right eye 10 years previously and glaucoma surgery 1 year previously presenting with 1 month of pain and frequent floaters in front of the right eye. On examination, the glass fragment was seen to lie free in the anterior chamber or migrate backwards through the pupil, remaining mobile on the inferior retinal surface when the patient was prone or supine, respectively. Results: The fragment was surgically removed. Conclusion: Late migration of glass intraocular foreign bodies is a rare clinical entity, and the exact mechanism causing the migration of intraocular glass remains controversial. Early intervention must be weighed against the hazards of removal and the necessity of close follow-up.
\end{abstract}

(C) 2015 S. Karger AG, Basel

\section{Background}

There are rare reports describing late spontaneous movement of glass intraocular foreign bodies (IOFBs). The present report describes the case of late recurrent migration of glass splinters from the vitreous cavity into the anterior chamber and the resultant additional anterior segment decompensation. 
Zhou et al.: Free Migration of Intraocular Glass in Aphakia after Glaucoma Surgery

\section{Case Report}

A 27-year-old man with a history of perforating injury to the right eye caused by an explosion of a glass beer bottle 10 years previously presented to the emergency department with 1 month of pain and frequent floaters in front of the right eye. All the glass in the anterior segment as well as the injured lens were removed. One year previously, he had undergone an Ahmed valve implantation in the right eye for glaucoma elsewhere. On examination, the glass fragment was seen to lie free in the anterior chamber (fig. 1a) or migrate backwards through the pupil, remaining mobile on the inferior retinal surface when the patient was prone or supine (fig. 1b), respectively. This was further examined with B-scan ultrasonography (fig. 1c). The fragment was surgically removed through a limbal incision, and corneal edema was alleviated.

\section{Discussion}

There are rare reports of late migration of glass fragments from the posterior to the anterior segment of the eye. Milkowski [1] presents cases of retained glass IOFBs near the retina, vitreous, and lens where complications did not develop. He also summarizes rare cases of migration of posterior IOFBs into the anterior chamber, causing corneal edema, cataract, and iridocyclitis. Gopal et al. [2] report on 8 eyes with glass IOFBs left behind without any untoward events during an average of 31 months of follow-up. In none of these eyes was any late anterior or posterior segment complication encountered. They hypothesized that preretinal fibrosis encapsulates a glass IOFB and provides additional security. In contrast, Ray et al. [3] show that the late migration of glass IOFBs can cause additional retinal pathologic conditions. However, late posterior segment migration that may be associated with glaucoma surgery, as in this case, must be rare.

The exact mechanism causing the migration of intraocular glass remains unclear. It is difficult to explain the forward movement, and various factors need to be considered. It could be influenced by head and eye movements, repeated trauma, or sporting activities [4]. In cases of aphakia, splinters can more easily move from the vitreous cavity to the anterior chamber. In the present case, there were large openings in the posterior capsule following the initial injury. The fact that glass splinters started to appear in the anterior chamber and caused corneal edema 1 year after the glaucoma surgery is probably due to the progressive liquefaction of the vitreous. Inflammation, fibrin reaction, or changes to the composition of the aqueous humor caused by tube insertion may contribute to the tendency to vitreous liquefaction $[5,6]$.

Glass IOFBs can pose special challenges. The irregular shape and sharpness of the edges of glass IOFBs can create further ocular injury during removal. Thus, early intervention must be weighed against the hazards of removal and the necessity of close follow-up.

\section{Statement of Ethics}

Informed consent has been obtained from the subject and the study protocol has been approved by the institute's committee on human research. 
Zhou et al.: Free Migration of Intraocular Glass in Aphakia after Glaucoma Surgery

\section{Disclosure Statement}

None of the authors have any conflicts of interests regarding the material presented in this paper.

\section{References}

1 Milkowski S: Rare case of spontaneous migration of intraocular glass foreign body 21 years after injury (in Polish). Wiad Lek 1978;31:1065-1069.

-2 Gopal L, Banker A, Deb N, Badrinath SS, Sharma T, Parikh SN, Shanmugham MP, Bhende PS, Das D, Mukesh BN: Management of glass intraocular foreign bodies. Retina 1998;18:213-220.

-3 Ray S, Friberg TA, Beatty RR, Loewenstein J: Late posterior migration of glass intraocular foreign bodies. Arch Ophthalmol 2004;122:923-926.

-4 Saar I, Raniel J, Neumann E: Recurrent corneal oedema following late migration of intraocular glass. Br J Ophthalmol 1991;75:188-189.

-5 Minckler DS, Francis BA, Hodapp EA, Jampel HD, Lin SC, Samples JR, Smith SD, Singh K: Aqueous shunts in glaucoma: a report by the American Academy of Ophthalmology. Ophthalmology 2008;115:1089-1098.

-6 Lee EK, Yun YJ, Lee JE, Yim JH, Kim CS: Changes in corneal endothelial cells after Ahmed glaucoma valve implantation: 2-year follow-up. Am J Ophthalmol 2009;148:361-367.
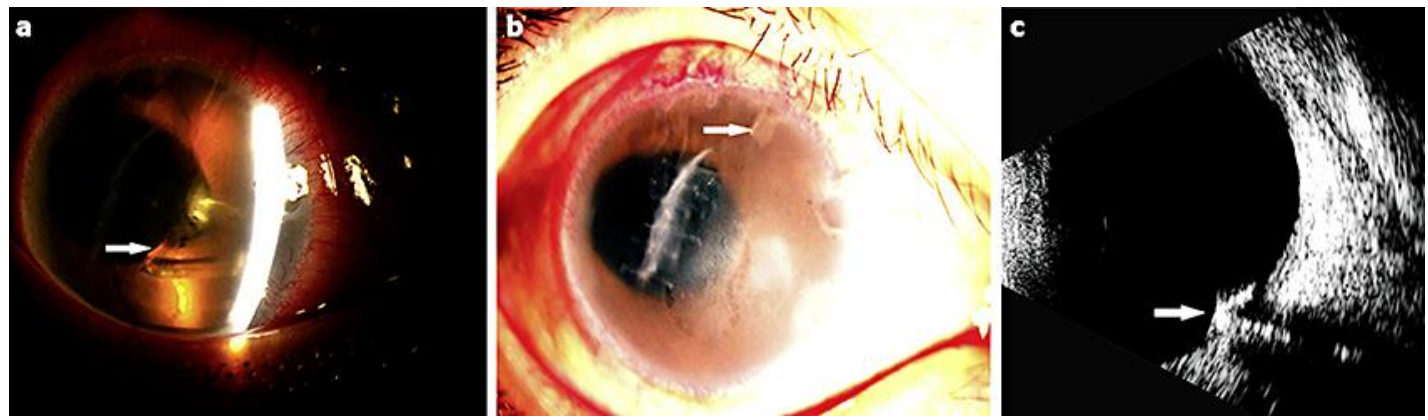

Fig. 1. Evaluation of the right eye. a Digital slit-lamp image showing the glass fragment in the anterior chamber in the prone position (arrow). $\times 10$. b Digital slit-lamp image showing no glass fragment in the anterior chamber in the supine position as well as the Ahmed valve (arrow). c B-scan ultrasonography in the supine position showing the glass fragment (arrow) on the retinal surface. 